\title{
Self-Similarity Properties of Natural Images Resemble Those of Turbulent Flows
}

\author{
Antonio Turiel, Germán Mato, and Néstor Parga* \\ Departamento de Física Teórica, Universidad Autónoma de Madrid, Cantoblanco, 28049 Madrid, Spain \\ Jean-Pierre Nadal \\ Laboratoire de Physique Statistique de l'E.N.S., ${ }^{\dagger}$ Ecole Normale Supérieure, 24, rue Lhomond, F-75231 Pairs Cedex 05, France \\ (Received 4 September 1997)
}

\begin{abstract}
We show that the statistics of an edge type variable in natural images exhibits self-similarity properties which resemble those of local energy dissipation in turbulent flows. Our results show that selfsimilarity and extended self-similarity hold remarkably for the statistics of the local edge variance, and that the very same models can be used to predict all of the associated exponents. These results suggest using natural images as a laboratory for testing more elaborate scaling models of interest for the statistical description of turbulent flows. The properties we have exhibited are relevant for the modeling of the early visual system: They should be included in models designed for the prediction of receptive fields. [S0031-9007(97)05190-9]
\end{abstract}

PACS numbers: 42.30.Yc, 05.70.Jk, 42.66.Lc, 47.27.Gs

The existence of self-similarity (SS) is well known in both natural images [1] and fully developed turbulence [2]. Quite recently, there has been an increase of interest in both fields. In turbulent flows, the notion of "extended self-similarity" (ESS) [3-5] has been introduced, and several models proposed predicting correctly the relevant SS exponents from only one or two parameters [6,7]. Our main motivation for studying the statistics of natural images is its relevance for the modeling of the early visual system. In particular, the epigenetic development could lead to the adaptation of visual processing to the statistical regularities in the visual scenes [8-13]. Most of these predictions on the development of receptive fields have been obtained using a Gaussian description of the environment contrast statistics. However, non-Gaussian properties such as the ones found by $[14,15]$ could be important. To gain further insight into non-Gaussian aspects of natural scenes we investigate whether they exhibit the rich structure found in turbulent flows.

Scaling properties of natural images have been studied by several authors. They have found $[1,16,17]$ that the power spectrum of luminosity contrast follows a power law of the form $S(f) \propto \frac{1}{|f|^{2-\eta}}$, although the value of $\eta$ can have rather large fluctuations [18]. The magnitude of these fluctuations depends on the diversity of the images in the data set. A more detailed-although differentanalysis of the scaling properties of image contrast was done by $[14,15]$ who also noted analogies with the statistics of turbulent flows. Additional luminosity analysis was also done by Ruderman [19], providing some evidence of multiscaling behavior. There is, however, no model to explain the intriguing scaling behavior observed.

However, in turbulent fluids the unpredictable character of signals has led to a large amount of effort in order to develop statistical models (see, e.g., Ref. [20]). Qualitative and quantitative theories of the statistical properties of fully developed turbulence elaborate on the original ar- gument of Kolmogorov [2]. The cascade of energy from one scale to another is described in terms of local energy dissipation per unit mass within a box of linear size $r$. This quantity, $\epsilon_{r}$, is given by

$$
\epsilon_{r}(\mathbf{x}) \propto \int_{\left|\mathbf{x}-\mathbf{x}^{\prime}\right|<r} d \mathbf{x}^{\prime} \sum_{i j}\left[\partial_{i} v_{j}\left(\mathbf{x}^{\prime}\right)+\partial_{j} v_{i}\left(\mathbf{x}^{\prime}\right)\right]^{2},
$$

where $v_{i}(\mathbf{x})$ is the $i$ th component of the velocity at point $\mathbf{x}$. Self-similarity will hold if, for some range of scales $r$, one finds the scaling relation,

$$
\left\langle\epsilon_{r}^{p}\right\rangle \propto r^{\tau_{p}},
$$

where $\left\langle\epsilon_{r}^{p}\right\rangle$ denotes the $p$ th moment of the energy dissipation that is the average of $\left[\epsilon_{r}(\mathbf{x})\right]^{p}$ over all possible values of $\mathbf{x}$. In fluid dynamics this property holds in the so-called "inertial range" [20]. A more general scaling relation, called extended self-similarity, has been found to be valid in a much larger scale domain, even if the inertial range does not exist $[3,4]$. This scaling can be defined by

$$
\left\langle\epsilon_{r}^{p}\right\rangle \propto\left\langle\epsilon_{r}^{q}\right\rangle^{\rho(p, q)},
$$

where $\rho(p, q)$ is the ESS exponent of the $p$ th moment with respect to the $q$ th moment. Notice that if SS holds then $\tau_{p}=\tau_{q} \rho(p, q)$. In the following we will refer all the moments to $\left\langle\epsilon_{r}^{2}\right\rangle$.

The basic field in turbulence is the velocity from which one defines the local energy dissipation. The largest contributions to $\epsilon_{r}$ come from abrupt changes in velocities. For images, the basic field is the contrast $c(\mathbf{x})$ that we define as the difference between the luminosity and its average. A natural candidate for a variable analog to the local energy dissipation is a quantity which takes its largest contributions from the places where large changes in contrast occur. This is precisely a measure of the existence of edges below the scale under consideration. Edges are indeed well known to be very important in 
characterizing images [21]. A recent numerical analysis suggests that natural images are composed of statistically independent edges [22].

We choose to study two variables, defined at position $\mathbf{x}=\left(x_{1}, x_{2}\right)$ and at scale $r$. The variable $\epsilon_{h, r}(\mathbf{x})$ takes contributions from edges transverse to a horizontal segment of size $r$,

$$
\epsilon_{h, r}(\mathbf{x})=\left.\frac{1}{r} \int_{x_{1}}^{x_{1}+r}\left(\frac{\partial c\left(\mathbf{x}^{\prime}\right)}{\partial y}\right)^{2}\right|_{\mathbf{x}^{\prime}=\left\{y, x_{2}\right\}} d y .
$$

A vertical variable $\epsilon_{v, r}(\mathbf{x})$ is defined similarly from an integration over the vertical direction. From here we see that $\epsilon_{l, r}(\mathbf{x})(l=h, v)$ is the local linear edge variance along the direction $l$ at scale $r$.

We have analyzed the scaling properties of the local linear edge variances in a set of 45 images taken in the wood of $256 \times 256$ pixels each (see [15] for technical details concerning these images). With these data, one can explore scales up to $r \sim 64$ pixels.

First, we show that SS holds in a range of scales $r$ with exponents $\tau_{h, p}$ and $\tau_{v, p}$. This is illustrated in Fig. 1, where the logarithm of the moments of the vertical and horizontal edge variances [as defined in Eq. (4) for the horizontal case] is plotted as a function of $\ln r$. Next, we test ESS. The results are shown in Fig. 2, where a linear behavior of $\ln \left\langle\epsilon_{l, r}^{p}\right\rangle$ vs $\ln \left\langle\epsilon_{l, r}^{2}\right\rangle$ is observed in both the horizontal $(l=h)$ and the vertical $(l=v)$ directions. One can see that ESS is valid in a wider range than SS. This is similar to what is found in turbulence, where this property has been used to obtain a more accurate estimation of the exponents of the structure functions (see, e.g., [23]). The horizontal and vertical exponents $\rho_{h}(p, 2)$ and $\rho_{v}(p, 2)$, estimated with a least squares regression, are shown in Fig. 3 as a function of $p$. From Figs. 13 , one sees that the horizontal and vertical directions have similar statistical properties, which was not expected (e.g., trees tend to increase luminosity correlations in the vertical direction). The SS exponents differ, as can be seen in Fig. 1. What is even more surprising is that ESS not only holds for the statistics in both directions, but it does it with the same ESS exponents, i.e., $\rho_{h}(p, 2) \sim$ $\rho_{v}(p, 2)$, within our numerical accuracy.

Let us now consider scaling models to predict the $p$ dependence of the ESS exponents $\rho_{l}(p, 2)$. Since ESS holds, the SS exponents $\tau_{l, p}$ can be obtained from the $\rho_{l}(p, 2)$ 's by measuring $\tau_{l, 2}$. The simplest scaling hypothesis is that, for a random variable $\epsilon_{r}(\mathbf{x})$ observed at the scale $r$ [such as $\epsilon_{l, r}(\mathbf{x})$ ], its probability distribution $\bar{P}_{r}\left[\epsilon_{r}(\mathbf{x})=\epsilon\right]$ can be obtained from any other scale $L$ by

$$
\bar{P}_{r}(\epsilon)=\frac{1}{\alpha(r, L)} \bar{P}_{L}\left(\frac{\epsilon}{\alpha(r, L)}\right) .
$$

From this, one derives that $\alpha(r, L)=\left[\frac{\left\langle\epsilon_{r}^{p}\right\rangle}{\left\langle\epsilon_{L}^{p}\right\rangle}\right]^{1 / p}$ for any $p$, and that $\rho(p, 2) \propto p$. If SS holds, then $\tau_{p} \propto p$ : For turbulent flows this corresponds to the Kolmogorov predic-

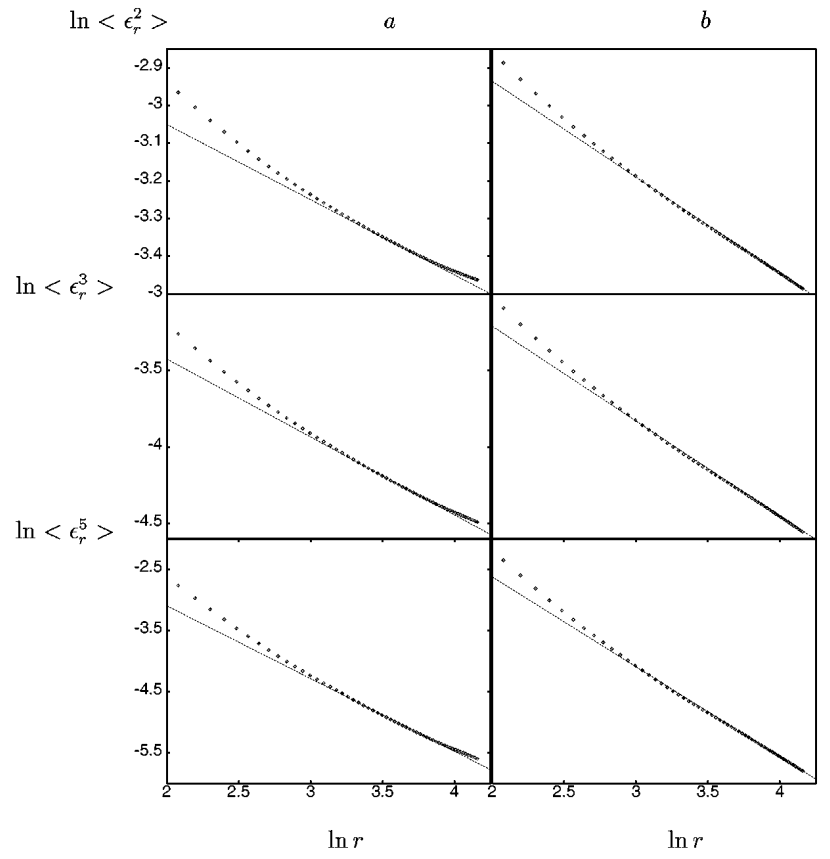

FIG. 1. Test of SS. We plot $\ln \left\langle\epsilon_{l, r}^{p}\right\rangle$ vs $\ln r$ for $p=2,3$, and 5 , and for $r$ from 8 to 64 pixels. (a) Horizontal direction, $l=h$; (b) vertical direction, $l=v$. The relative error is uniform and about $8 \%$. The value of the SS exponents $\tau_{p}$ extracted from the large $r$ behavior are $\tau_{h, 2}=-0.20 \pm 0.01$, $\tau_{h, 3}=-0.51 \pm 0.02$, and $\tau_{h, 5}=-1.19 \pm 0.06$ for the horizontal direction, and $\tau_{v, 2}=-0.26 \pm 0.04, \tau_{v, 3}=-0.62 \pm$ 0.03 , and $\tau_{v, 5}=-1.47 \pm 0.06$ for the vertical direction. The solid lines are the slope given by these exponents. This linear behavior does not hold at small $r$. A numerical analysis indicates that it is a finite resolution effect although it could be masking a different, small $r$ regime. There is also an upper bound that has prevented us from going beyond $r \sim 64$.

tion for the SS exponents [2]. The nonlinear behavior observed in Fig. 3 shows that this naive scaling is violated (this is similar to what was observed in turbulence [24], where the nonlinear behavior was interpreted as evidence of the multifractal character of the turbulent flows [25]). This discrepancy becomes more dramatic if Eq. (5) is expressed in terms of a normalized variable. Taking $\epsilon_{r}^{\infty}=\lim _{p \rightarrow \infty}\left\langle\epsilon_{r}^{p+1}\right\rangle /\left\langle\epsilon_{r}^{p}\right\rangle$, the new variable is defined as $f_{r}=\epsilon_{r} / \epsilon_{r}^{\infty}$. If $P_{r}(f)$ is the distribution of $f_{r}$ the scaling relation, Eq. (5), reads $P_{r}(f)=P_{L}(f)$. That this identity does not hold can be observed in Fig. 4. A way to generalize this scaling hypothesis is to say that, instead of having one value of $\alpha$ as in (5), every value of $\alpha$ contributes with a given weight. One then has

$$
P_{r}(f)=\int G_{r L}(\ln \alpha) \frac{1}{\alpha} P_{L}\left(\frac{f}{\alpha}\right) d \ln \alpha .
$$

This scaling relation has been first introduced in the context of turbulent flows $[6,7,26,27]$. One can see that Eq. (6) is an integral representation of ESS with general (not necessarily linear) exponents. Once a kernel $G_{r L}$ is chosen the $\rho(p, 2)$ 's can be predicted. 


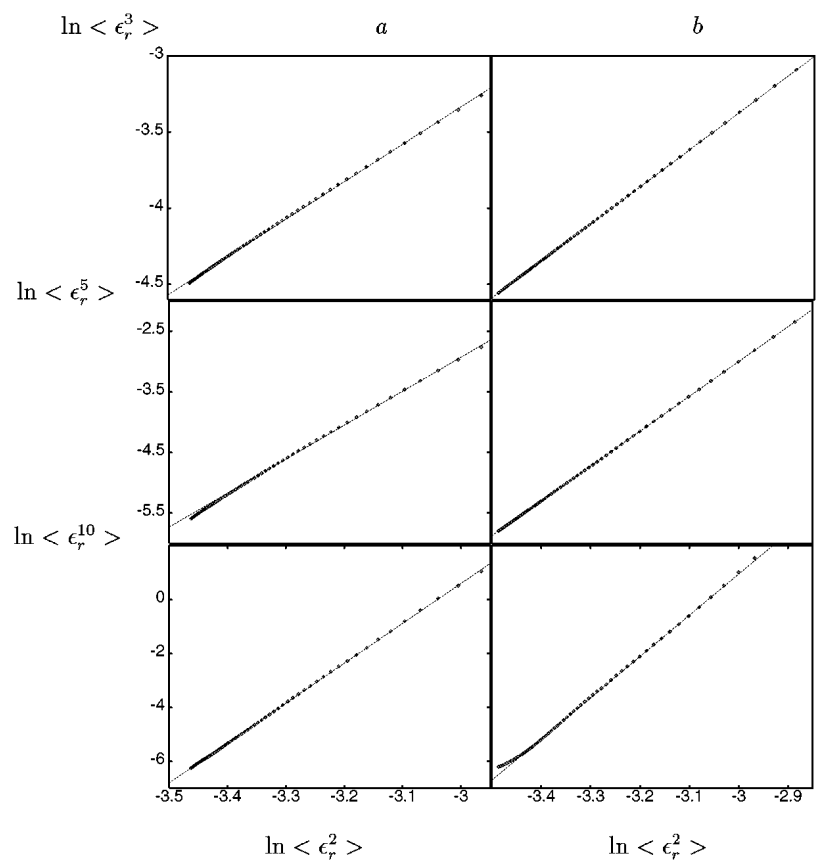

FIG. 2. Test of ESS. We plot $\ln \left\langle\epsilon_{l, r}^{p}\right\rangle$ vs $\ln \left\langle\epsilon_{l, r}^{2}\right\rangle$ for $p=3$, 5, and 10. Data correspond to scales from $r=8$ to $r=64$ pixels. The effect of finite size effects can again be observed for $r$ close to 64 pixels. (a) Horizontal direction, $l=h$; (b) vertical direction, $l=v$. The solid lines are the slope given by the calculated exponents $\rho(p, 2)$.

The difference between Eqs. (5) and (6) can also be phrased in terms of multiplicative processes [28,29]. Instead of $f_{r} \sim f_{L}$, we now have $f_{r} \sim \alpha f_{L}$, where the fac-

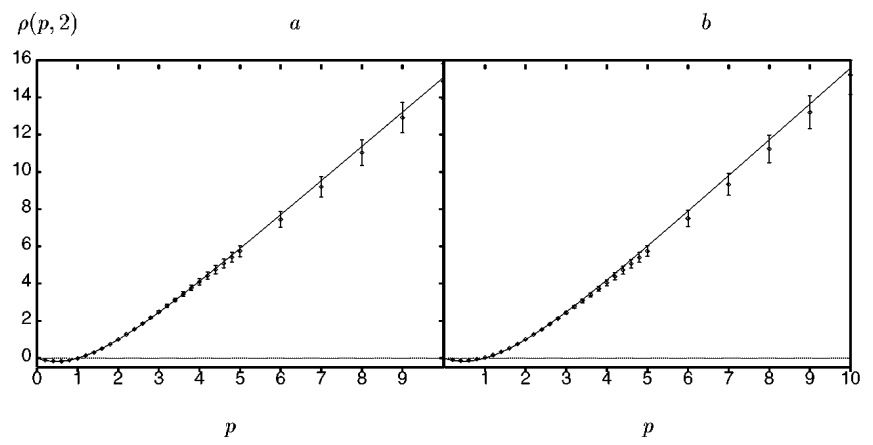

FIG. 3. ESS exponents $\rho(p, 2)$, for the vertical and horizontal variables. Each value of $\rho_{l}(p, 2)$ was obtained by a linear regression of $\ln \left\langle\epsilon_{l, r}^{p}\right\rangle$ vs $\ln \left\langle\epsilon_{l, r}^{2}\right\rangle$ for distances $r$ between 8 and $64(l=v, h)$. (a) Horizontal direction, $\rho_{h}(p, 2)$; (b) vertical direction, $\rho_{v}(p, 2)$. The solid line represents the fit with the SL model. The best fit is obtained with $\beta_{v} \sim \beta_{h} \sim 0.50$. The error bars $b_{p}$ have been estimated by dividing the 45 images into 9 groups, evaluating $\rho_{l}(p, 2)$ for each of them, and computing the dispersion of these values. The errors grow as $p$ increases. This is because moments of higher order are sensitive to the tail of the distribution of the local edge variance. The fit is such that the following average quadratic error, $E=\sum_{p} \frac{\left[\rho(p, 2)_{\exp }-\rho(p, 2)_{\mathrm{th}}\right]^{2}}{b_{p}}$, is minimized. We have checked that a Gaussian data set of images does exhibit ESS although it cannot be explained by the SL model. tor $\alpha$ itself becomes a stochastic variable determined by the kernel $G_{r L}(\ln \alpha)$. Since the scale $L$ is arbitrary (scale $r$ can be reached from any other scale $r^{\prime}$ ), the kernel must obey a composition law. This stochastic variable at scale $r$ can then be obtained through a cascade of infinitesimal processes $G_{\delta} \equiv G_{r, r+\delta r}$.

Specific choices of $G_{\delta}$ define different models of ESS. The She-Leveque (SL) [6] model corresponds to a simple process such that $\alpha$ is 1 with some probability $1-s$ and is a constant $\beta$ with probability $s$. One can see that $s=\frac{1}{1-\beta^{2}} \ln \left(\frac{\left\langle f_{r+\delta r}^{2}\right\rangle}{\left\langle f_{r}^{2}\right\rangle}\right)$ and that this stochastic process yields a log-Poisson distribution for $\alpha$ [30]. It also gives ESS with exponents $\rho(p, q)$ that can be expressed in terms of a single parameter $(\beta)$ as follows [6]:

$$
\rho(p, q)=\frac{1-\beta^{p}-(1-\beta) p}{1-\beta^{q}-(1-\beta) q} .
$$

We have tested the model with the ESS exponents obtained with the image data set. The resulting fit for the SL model is shown in Fig. 3. Both the vertical and horizontal ESS exponents can be fitted with $\beta=0.50 \pm$ 0.03. More complex processes other than log-Poisson

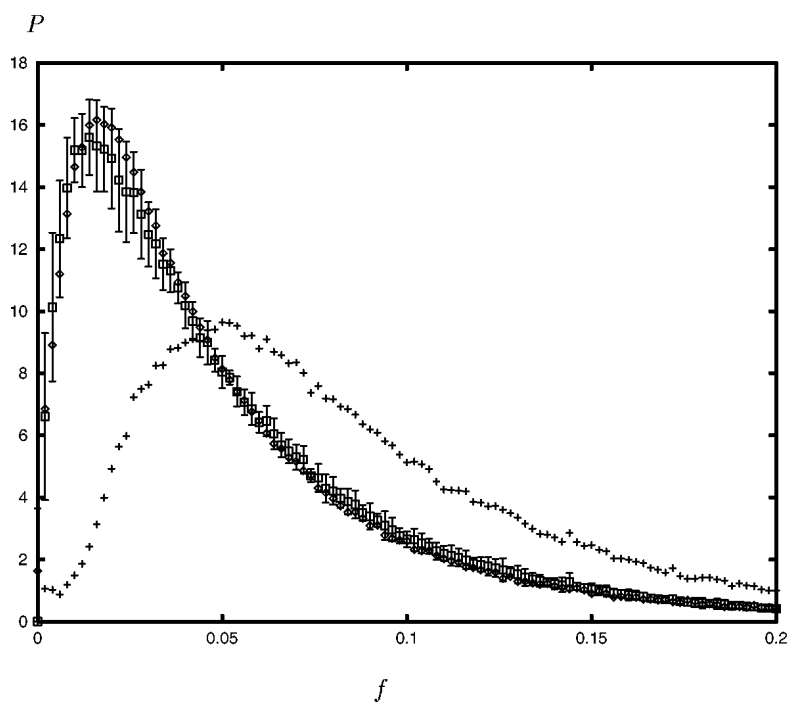

FIG. 4. Verification of the validity of the integral representation of ESS, Eq. (6) with a log-Poisson kernel, for horizontal local edge variance. The largest scale is $L=64$. Starting from the histogram $P_{L}(f)$ (crosses), and using a log-Poisson distribution with parameter $\beta=0.50$ for the kernel $G_{r L}$, Eq. (6) gives a prediction for the distribution at the scale $r=16$ (squares). This has to be compared with the direct evaluation of $P_{r}(f)$ (diamonds). Similar results hold for other pairs of scales. The error bars have been estimated as follows: The data set was divided in nine groups, as explained in the previous figure, and the histograms at the scales $L$ and $r$ were computed for each group. Then for each group the histogram at scale $L$ was used to obtain a prediction for the histogram at scale $r$. The differences between the predicted and computed values were squared and averaged over the groups. Its square root gives a measure of the error committed in the prediction, represented by the error bars. The test for the vertical case is as good as for the horizontal variable. 
distributions, involving more than one parameter, have also been studied. We have also tested the model proposed in [7]. For our data, the best fit appears to be with the SL model, which is the simplest nontrivial model.

The integral representation of ESS [Eq. (6)], can also be tested directly on the probability distributions $P_{r}(f)$ and $P_{L}(f)$ evaluated from the data. In Fig. 4 we show the prediction for the distribution at the scale $r$ obtained from the distribution at the scale $L$. No new parameter is needed for this.

The parameter $\beta$ has allowed us to obtain all the ESS exponents $\rho(p, 2)$. In order to obtain the SS exponents $\tau_{p}$ we need another parameter, e.g., $\tau_{2}$. Notice first that, for large $r, \epsilon_{r}^{\infty} \propto r^{\tau_{2} / 1-\beta} \equiv r^{-\Delta}$. From the definition of $\epsilon_{r}^{\infty}$, one sees that it is controlled by the tail of the distribution $\bar{P}_{r}(\epsilon)$. This implies that the most singular structure is the set of points where $\epsilon_{r}=\epsilon_{r}^{\infty}$. Now a standard argument on multifractal scaling (see, e.g., $[20,31])$ will relate the exponent $\Delta$ to the dimension $D_{\infty}$ of this most singular structure. One finds $D_{\infty}=d-\frac{\Delta}{1-\beta}$, where $d=2$ is the dimensionality of the problem. Since $\tau_{p}=\tau_{2} \rho(p, 2)$, a fit of $\tau_{p}$ determines $\Delta$. This was done for both the vertical and horizontal variables, obtaining $\Delta_{h}=0.4 \pm 0.2$ and $\Delta_{v}=0.5 \pm 0.2$ and leading to $D_{\infty, h}=1.3 \pm 0.3$ and $D_{\infty, v}=1.1 \pm 0.3$. The quoted errors are purely statistical, but other sources of errors (e.g., the onset of the SS behavior) reduce the accuracy. As a result, we can say that $D_{\infty, v} \sim D_{\infty, h} \sim 1$ : The most singular structures are almost one dimensional; this reflects the fact that the most singular manifold consists of sharp edges.

In conclusion, we insist on the main result of this work, which is the existence of nontrivial scaling properties for the local edge variances. This property appears very similar to the one observed in turbulence for the local energy dissipation. In fact, we have seen that the SL model predicts all of the relevant exponents and that, in particular, it describes the scaling behavior of the sharpest edges in the image ensemble. A similar analysis could be performed taking into account color or motion (analyzing video sequences). It would also be interesting to have a simple generative model of images whichapart from having the correct power spectrum as in [32] would reproduce the self-similar properties found in this paper.

We are grateful to Dan Ruderman for giving us his image data base. We thank Bernard Castaing for very stimulating discussions, and Zhen-Su She for a discussion on the link between the scaling exponents and the dimension of the most singular structure. We thank Roland Baddeley and Patrick Tabeling for fruitful discussions. We also acknowledge Nicolas Brunel for his collaboration during the early stages of this work. This work has been partly supported by the French-Spanish program, "Picasso," and an E.U. Grant.
*To whom correspondence should be addressed.

${ }^{\dagger}$ Laboratoire associé au C.N.R.S. (U.R.A. 1306, à l'ENS, et aux Universités Pairs IV et Pairs VII.

[1] D. J. Field, J. Opt. Soc. Am. 4, 2379-2394 (1987).

[2] A. N. Kolmogorov, Dokl. Akad. Nauk. SSSR 30, 301-305 (1941).

[3] R. Benzi, S. Ciliberto, C. Baudet, G. Ruiz Chavarria, and R. Tripiccione, Europhys. Lett. 24, 275-279 (1993).

[4] R. Benzi, S. Ciliberto, C. Tripiccione, C. Baudet, F. Massaioli, and S. Succi, Phys. Rev. E 48, R29 (1993).

[5] R. Benzi, S. Ciliberto, C. Baudet, and G. Ruiz Chavarria, Physica (Amsterdam) 80D, 385-398 (1995).

[6] Zhen-Su She and E. Leveque, Phys. Rev. Lett. 72, 336339 (1994).

[7] B. Castaing, J. Phys. II (France) 6, 105-114 (1996).

[8] H.B. Barlow, in Sensory Communication, edited by W. Rosenblith (MIT Press, Cambridge, MA, 1961), p. 217.

[9] S. B. Laughlin, Z. Naturforsch. 36, 910-912 (1981).

[10] J. H. van Hateren, J. Comput. Phys. A 171, 157-170 (1992).

[11] J. J. Atick, Network 3, 213-251 (1992).

[12] B. A. Olshausen and D. J. Field, Nature (London) 381, 607-609 (1996).

[13] R. Baddeley, Cogn. Sci. (to be published).

[14] D. Ruderman and W. Bialek, Phys. Rev. Lett. 73, 814 (1994).

[15] D. Ruderman, Network 5, 517-548 (1994).

[16] C. R. Carlson, Photogr. Sci. Eng. 22, 69-71 (1978).

[17] G. J. Burton and I. R. Moorhead, Appl. Opt. 26, 157-170 (1987).

[18] D. J. Tollhurst, Y. Tadmor, and Chao Tang, Ophthalmic Physiol. Opt. 12, 229-232 (1992).

[19] D. Ruderman (private communication).

[20] U. Frisch, Turbulence (Cambridge University Press, Cambridge, England, 1995).

[21] D. Marr, Vision (Freeman, San Francisco, 1982).

[22] A. J. Bell and T. J. Sejnowski, Vision Res. 37, 3327-3338 (1997).

[23] A. Arneodo et al., Europhys. Lett. 34, 411-416 (1996), and references therein.

[24] F. Anselmet, Y. Gagne, E. J. Hopfinger, and R. A. Antonia, J. Fluid Mech. 140, 63 (1984).

[25] G. Parisi and U. Frisch, in Turbulence and Predictability in Geophysical Fluid Dynamics, edited by M. Ghil, R. Benzi, and G. Parisi, Proceedings of the International School of Physics, E. Fermi (North-Holland Amsterdam, 1985), pp. 84-87.

[26] B. Castaing, Y. Gagne, and E. J. Hopfinger, Physica (Amsterdam) 46D, 177 (1990).

[27] B. Dubrulle, Phys. Rev. Lett. 73, 959-962 (1994).

[28] E. A. Novikov, Phys. Rev. E 50, R3303 (1994).

[29] R. Benzi, L. Biferale, A. Crisanti, G. Paladin, M. Vergassola, and A. Vulpiani, Physica (Amsterdam) 65D, 352-358 (1993).

[30] Zhen-Su She and E.C. Waymire, Phys. Rev. Lett. 74, 262-265 (1995).

[31] G. Paladin and A. Vulpiani, Phys. Rep. 156, 147-225 (1987).

[32] D. Ruderman, Vision Res. 37, 3385-3398 (1997). 